\title{
Metacontrast as a function of internal contour and target width
}

\author{
PATTI L. KELLY and ROBERT H. POLLACK \\ University of Georgia, Athens, Georgia 30602
}

\begin{abstract}
Extent of metacontrast was examined as a function of target internal contours and target thickness. Forty college students participated in a spatial forced-choice method of presentation with target-mask interstimulus intervals ranging from 1 to $100 \mathrm{msec}$. Masking decreased as both target thickness and target internal contour increased.
\end{abstract}

Metacontrast is the suppression of one visual stimulus by a second visual stimulus when the second stimulus falls on an adjacent but nonoverlapping area of the retina within a critical period of time. While many variables have been found to affect this phenomenon, the variable of primary interest in the present study was the spatial variable of degree of internal contour. From the classic study performed by Werner (1935) until recently, studies have indicated that the presence of internal contour within a target has decreased the target's susceptibility to masking. Pollack (1965) found that metacontrast was greatest when the contours of the test figure and the masking figure were parallel. Streicher and Pollack (1967) demonstrated that when contours of the target were not parallel with those of the mask, much less metacontrast occurred even when intercontour distances were comparable. Sherrick and Dember (1970), in examining the effect of area of the target on target detection in metacontrast, found that the degree of metacontrast was considerably less with nonhomogeneous targets, suggesting once again that degree and orientation of internal contours within the target were crucial variables in its susceptibility to metacontrast.

To assess further the effect of internal target contour on metacontrast, Cox and Dember (1970) varied quantitatively the amount of internal target contour by constructing targets that consisted of black and white pie-shaped segmented circles and varying the number of segments within each target. They found that as the number of internal target contours increased, the target's susceptibility to metacontrast decreased to such an extent that virtually no masking occurred for the 16segmented target. Further support for these results was found by Ellis and Dember (1971). Arand and Dember (1974) postulated that this relationship between susceptibility to masking and number of target segments should approach an asymptote at some value just preceding the points at which the limits of visual acuity would cause a segmented target to appear homogeneous. They found that whereas the function approached asymptote from 16 to 32 segments, more than 32 segments would be needed to produce the phenomenal effect of a complete circle.

In direct contradiction of all previous findings,
Lefton (1974) and Lefton and Griffin (1976) found that increasing the number of internal target segments increased susceptibility to metacontrast. Lefton (1974) found that a target with nine segments was masked more easily by a ring than a target with three segments. Although Lefton and Griffin (1976) supported Lefton's (1974) findings, some of the masks used in this study had internal projections that fitted exactly between the segments of the target, thus increasing the number of parallel contours and similarity of target and mask. Since Lefton and Griffin's (1976) controversial results can be explained in these terms alone, it was felt that no further empirical investigation was needed. However, close examination of Lefton's (1974) and Lefton and Griffin's (1976) targets revealed a small hole in the center of their targets. It was postulated that the introduction of this hole increased target-mask similarity by creating the subjective impression of an inner circle within the target, thereby increasing the susceptibility of the target to masking with rings. Not only did this hole serve to increase target-mask similarity, but more important, it eliminated the presence of intersecting contours within the target, thereby decreasing the overall amount of internal target contour. The present investigation was undertaken to assess systematically the effects of this hole on metacontrast.

While attempting to replicate Lefton's (1974) study, it was found that Lefton (1974) used scotopic luminance levels of $.12 \mathrm{~cd} / \mathrm{m}^{2}(.035 \mathrm{fL})$ when presenting his targets and allowed his subjects to adapt to ambient room illumination for only $5 \mathrm{~min}$. Since Lefton and Newman (1976) found that a scotopic luminance level of $.25 \mathrm{~cd} / \mathrm{m}^{2}$ produced not only a definite increase in metacontrast, but also extreme difficulty in detecting the targets at all, the present investigation employed Ellis and Dember's (1971) luminance level of $34.258 \mathrm{~cd} / \mathrm{m}^{2}$ (10 fL) because it was definitely photopic. Given that the total visual display subtended less than $5 \mathrm{deg}$ of visual angle, an area more densely populated with photopic than scotopic receptors, scotopic illumination would have made detection and resolution of both targets and masks difficult. According to Lefton (1974), luminance level should not have affected results dependent upon number of target segments. 


\section{HOLE SIZE $(\mathrm{mm})$}

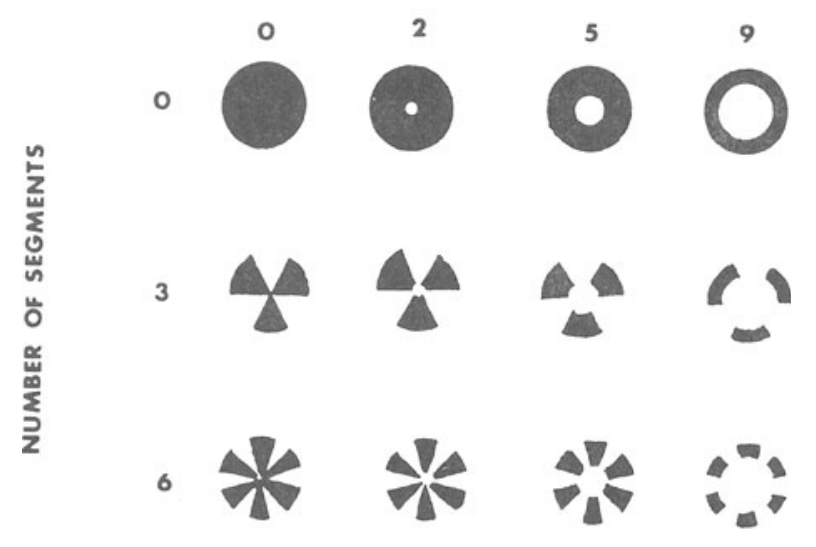

TARGETS

Figure 1. Targets used, closely resembling those used by Lefton (1974).

\section{METHOD}

\section{Subjects}

Forty undergraduate students (20 males and 20 females) at the University of Georgia served as volunteer subjects, whose participation was in partial fulfillment of the requirements of an introductory psychology course. All subjects had corrected or normal vision of 20/25 as measured by the Master Orthorator, and no subject with tinted contact lenses or tinted eyeglasses was allowed to participate.

\section{Stimulus Figures}

The targets consisted of disks drawn with black ink on white cardboard. These disks varied along two dimensions: (1) number of segments within the disks (no segments, three segments, or six segments) and (2) size of an inner circle separating the points of the segments (no hole, a 2-mm hole, a 5-mm hole, or a 9-mm hole). The segmented conditions were identical to those used by Lefton (1974), in that they appeared similar to a windmill, with alternating black and white segments, each of the same angle. All targets were $13 \mathrm{~mm}$ in diameter, and, at a viewing distance of $1,270 \mathrm{~mm}$, the center-to-center distance between the masks subtended a visual angle of $4 \mathrm{deg}$. (See Figure 1.) The masks consisted of black rings drawn on white cardboard with outer diameters of $26 \mathrm{~mm}$ and inner diameters of $13 \mathrm{~mm}$. These dimensions produced targets that subtended $.6 \mathrm{deg}$ of visual angle, as did Lefton's (1974) targets.

Two masking rings appeared on each trial, one to the right and one to the left. These rings were drawn so that the inner diameter of each ring subtended $.6 \mathrm{deg}$ of visual angle. The targets were concentric to the rings, but they did not overlap. The visual angle subtended by the masks was identical to the visual angle subtended by Lefton's (1974) masks.

\section{Apparatus}

A three-channel tachistoscope, Scientific Prototype Model GB, was used for all presentations. Intensities of all channels were adjusted to provide Macbeth illuminometer readings of $10 \mathrm{fL}$ from the background used in this study.

\section{Procedure}

Upon introduction to the laboratory, subjects were adapted for $5 \mathrm{~min}$ to the background illumination of the tachistoscope.
During adaptation and during intertrial and interstimulus intervals, a fixation field that consisted of a small black dot on a white card (equidistant from all edges of the card) was present.

Each of the 12 targets was presented binocularly in the following sequence: fixation, target, interstimulus interval (fixation dot present), masking rings, and then the fixation field. Mask and target durations were $200 \mathrm{msec}$ and $10 \mathrm{msec}$, respectively. Interstimulus interval (ISI) varied from 0 to $100 \mathrm{msec}$ in 10 -msec steps.

On each trial, both masking rings were presented with the target falling within the center of one of the masking rings, concentric to it, but nonoverlapping. Subjects were required to make a spatial forced-choice decision as to the side of the field on which the target had been presented. The ISI necessary for the detection of each target was obtained using the converging method of limits. Each target was exposed to each subject for eight trial series. Order of target presentation was randomized across subjects.

Each subject reported for two experimental sessions (scheduled at the same time of day). Each experimental session lasted approximately $45 \mathrm{~min}$, during which the subject was exposed to 6 of the 12 targets until he/she had viewed the entire set.

\section{RESULTS}

The number of correct detections for each target for each subject was computed and entered into a three-way analysis of variance (number of target segments by hole size by ISI). (See Table 1.) All main effects and interactions were found to be significant beyond the .01 level. The significance of the three-way interaction was due in part to a ceiling effect produced by increasing the duration of the ISI and in part to an interaction between hole size and number of segments within the target. For the most part, as hole size increased, the effect of the number of segments decreased.

All two-way interactions with ISI were the result of the ceiling effect. (See Figures 2 and 3.) As the duration of the ISI increased, masking decreased independently of both the size of the hole within the target and the number of segments within the target. The significance of the two-way interaction between hole size and segments is accounted for not only by the fact that as the hole size increased, the more similar the

Table 1

Results of Analysis of Variance

\begin{tabular}{lrrrr}
\hline \multicolumn{1}{c}{ Source } & \multicolumn{1}{c}{ SS } & df & MSe & F \\
\hline Subjects (S) & 236.48 & 39 & & \\
SEG & 54.22 & 3 & 18.073 & 18.019 \\
SEG by S & 117.39 & 117 & 1.003 & \\
HS & 60.94 & 2 & 30.470 & 25.057 \\
HS by S & 94.86 & 78 & 1.220 & \\
ISI & 611.88 & 5 & 122.376 & 68.866 \\
ISI by S & 346.51 & 195 & 1.777 & \\
SEG by HS & 28.27 & 6 & 4.712 & 4.647 \\
SEG by HS by S & 237.37 & 234 & 1.010 & \\
SEG by ISI & 45.05 & 15 & 3.000 & 7.116 \\
SEG by ISI by S & 247.00 & 585 & .422 & \\
HS by ISI & 72.99 & 10 & 7.299 & 13.128 \\
HS by ISI by S & 216.71 & 390 & .556 & \\
SEG by HS by ISI & 55.72 & 30 & 1.857 & 4.781 \\
Residual & 454.48 & 1170 & .388 & \\
Total & 2879.87 & 2879 & 1.135 & \\
\hline
\end{tabular}




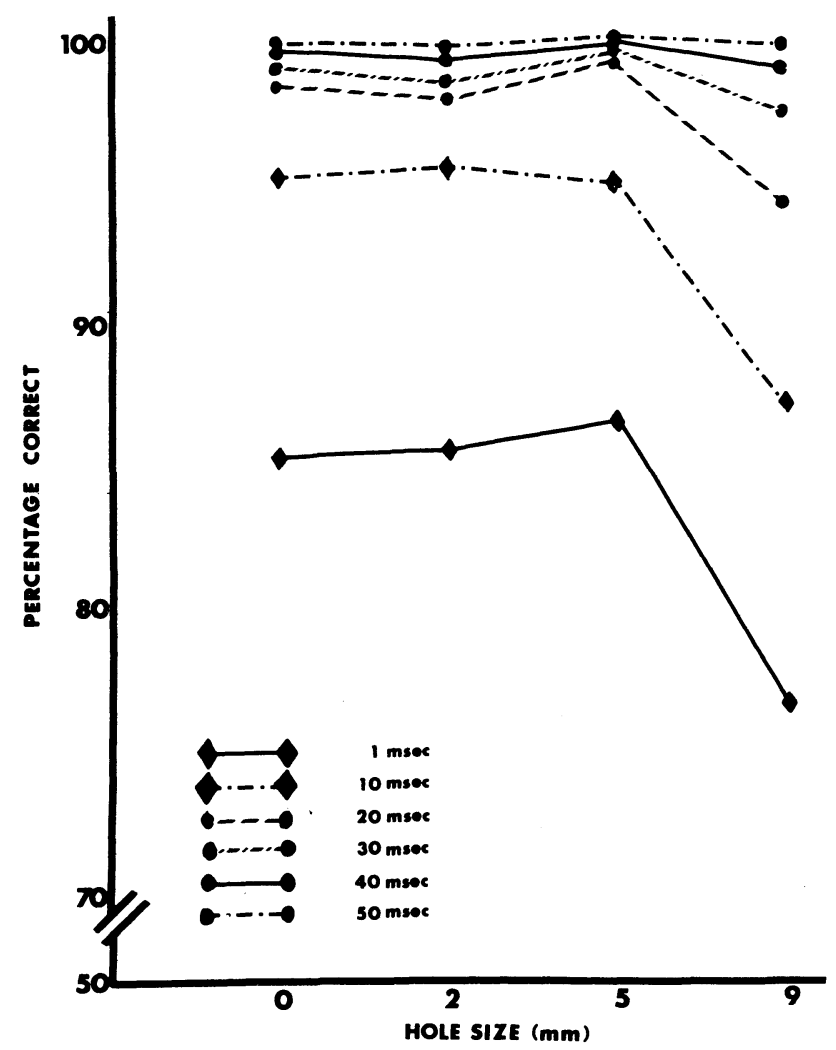

Figure 2. Magnitude of masking as a function of hole size for each interstimulus interval.

target became to the mask, but also by the fact that as hole size increased, the contours of the segments within the target became more closely parallel, thereby increasing the susceptibility of the target to metacontrast.

The ceiling effect produced by increases in the duration of the ISI was such that there was effectively no masking with ISIs greater than $50 \mathrm{msec}$, and asymptote was approached with ISIs of $30 \mathrm{msec}$. Conversely, as expected, the shorter the duration of the ISI, the greater was the amount of metacontrast, a finding in agreement with all previous research on the effect of ISI and metacontrast.

The effect of hole size on target detection was in the predicted direction (i.e., generally speaking, as the size of the hole increased, susceptibility to masking also increased). For the most part, within each segmented condition, a target with no hole exhibited less susceptibility to masking than did a target with a 9-mm hole. (See Figure 4.) Despite the presence of increased contour in the segmented targets, the segmented targets with internal holes were less resistant to masking than were homogeneous targets with holes of the same size. The presence of this internal hole eliminates intersecting contours within the target. Therefore, any contributions that the hole makes to target-mask similarity may be outweighed by its elimination of contour.

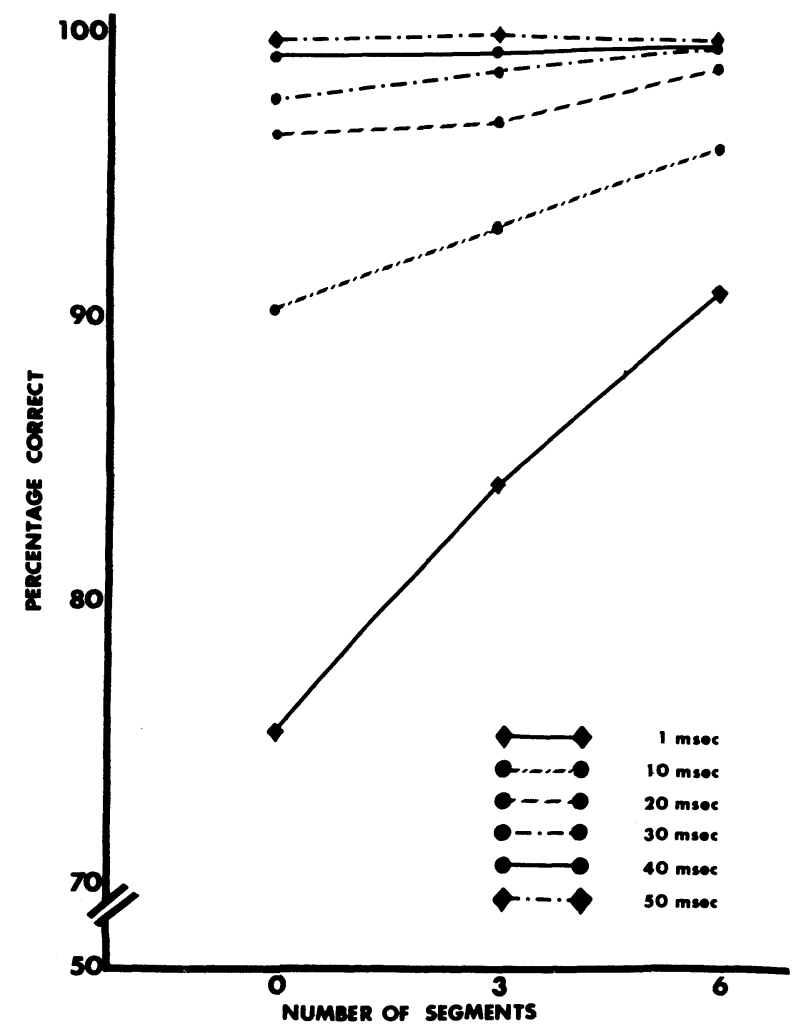

Figure 3. Magnitude of masking as a function of number of segments for each interstimulus interval.

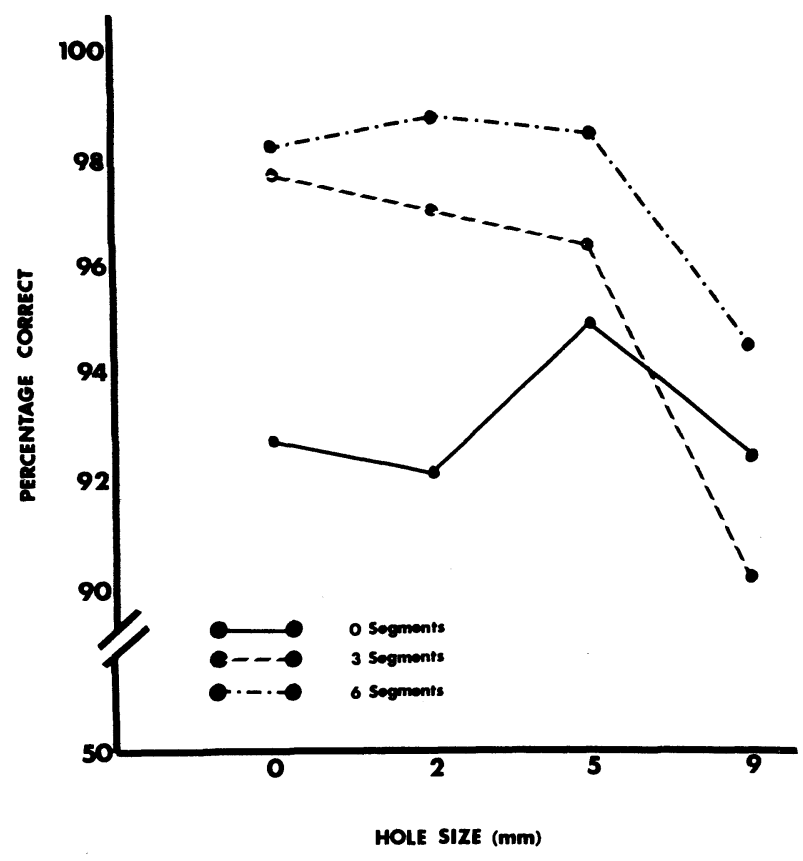

Figure 4. Magnitude of masking as a function of hole size for each segment condition. 


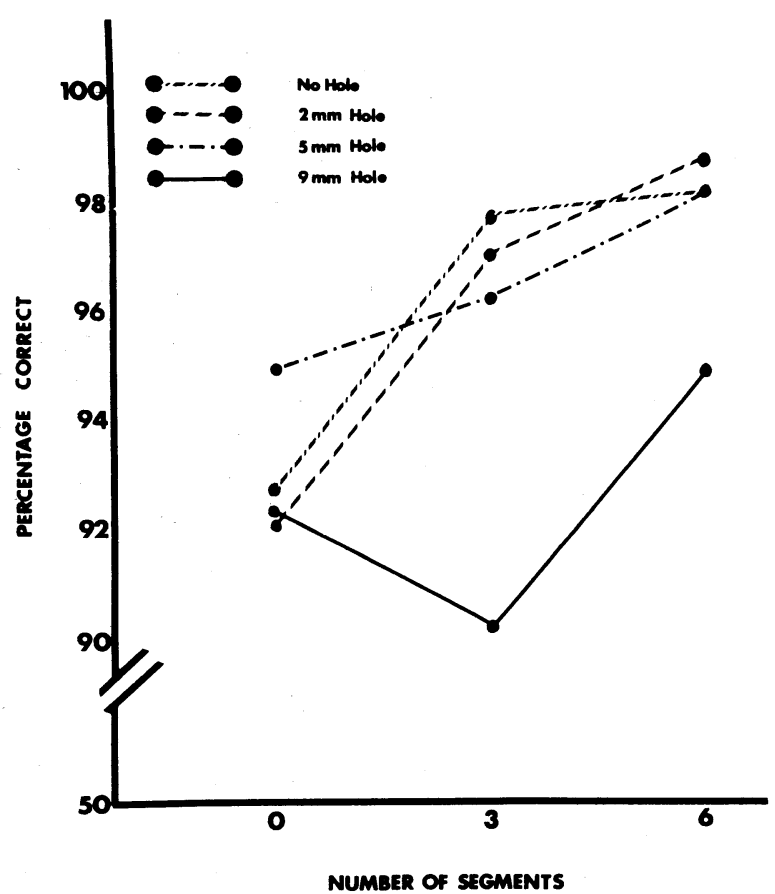

Figure 5. Magnitude of masking as a function of number of segments for each hole size condition.

The general effect of increasing the amount of contour within the target was to decrease the target's susceptibility to metacontrast. (See Figure 5.) In three of the four hole conditions (no hole, 2-mm hole, and 5-mm hole conditions), as targets increased from no segments to three segments to six segments, there was a general decrease in susceptibility to metacontrast.

\section{DISCUSSION}

The present study attempted to assess the effects of target mask similarity and degree of internal target contour as possible confounds in Lefton's (1974) and Lefton and Griffin's (1976) controversial results. The procedures used in this study were chosen to replicate as closely as possible those used by Lefton (1974). The experiment's only methodological point of departure from Lefton's (1974) design was in the luminance level of presentation, for the simple reason that the targets were not visible at Lefton's (1974) luminance level.

In agreement with research prior to Lefton's (1974) study, increases in the amount and quality of internal target contour were found to increase the difficulty with which these targets were masked. These results are in direct contradiction to those of Lefton (1974) and strongly support the results of Ellis and Dember (1971). Furthermore, the results of the present study indicate that the size of the hole within the target significantly decreased the number and character of the intersecting contours such that the larger the hole, the more easily the target was masked.

It should also be noted that the luminance levels used in the present study were higher than those used by Lefton (1974), but identical to those employed by Ellis and Dember (1971). Whereas it is also possible that the present results reflect a difference in the amount of light used (i.e., Lefton used transmitted light, whereas the present study used reflected light), a later publication of Lefton's (Lefton \& Newman, 1976) introduces the possibility that his 1974 luminance levels, as well as those of the 1976 study (Lefton \& Griffin, 1976), were too low to assess the effects of contour.

In conclusion, the degree and quality of contour within a target significantly affect that target's susceptibility to metacontrast by influencing such factors as target-mask similarity and the amount of intersecting or parallel contour present. In general, as the amount of contour increases within a target, its susceptibility to metacontrast decreases. It is also suggested that earlier discrepancies in conclusions regarding the role of internal target contour in metacontrast may be due in part to the introduction of confounding variables such as target-mask similarity.

\section{REFERENCES}

Arand, D., \& Dember, W. N. Masking effectiveness and number of segments in the masking ring. Bulletin of the Psychonomic Society, 1974, 3, 127-128.

Cox, S. I., \& Dember, W. N. Backward masking of visual targets with internal contours. Psychonomic Science, 1970, 19, 255-256.

Ellis, D., \& Dember, W. N. Backward masking of visual targets with internal contours: A replication. Psychonomic Science, 1971, 22, 91-92.

LEFTON, L. A. Internal contours, intercontour distance, and interstimulus interval: The complex interaction in metacontrast. Journal of Experimental Psychology, 1974, 103, 891-895.

Lefton, L. A., \& Griffin, J. P. Metacontrast with internal contours: More evidence for monotonic functions. Bulletin of the Psychonomic Society, 1976, 7, 29-32.

Lefton, L. A., \& Newman, Y. Metacontrast and paracontrast: Both photopic and scotopic luminance levels yield monotones. Bulletin of the Psychonomic Society, 1976, 8, 435-438.

Pollack, R. H. Effects of figure-ground contrast and contour orientation on figural masking. Psychonomic Science, 1965, 2 , 369-370.

Sherrick, M. F., \& Dember, W. N. Visual backward masking and the area-detection relation. Psychonomic Science, 1970, 19, 127-128.

Streicher, H. W., \& Pollack, R. H. Backward figural masking as a function of intercontour distance. Psychonomic Science, 1967, 7, 69-70.

WERNER, H. Studies in contour: I. Quantitative analysis. American Journal of Psychology, 1935, 47, 40-64.

(Received for publication April 16, 1981.) 